\title{
Solid-phase laser-induced forward transfer of variable shapes using a liquid-crystal spatial light modulator
}

\author{
R. Pohl ${ }^{1}$ • M. Jansink ${ }^{1}$ - G. R. B. E. Römer' ${ }^{1}$ A. J. Huis in 't Veld ${ }^{1}$
}

Received: 29 January 2015/Accepted: 3 May 2015/Published online: 12 May 2015

(c) The Author(s) 2015. This article is published with open access at Springerlink.com

\begin{abstract}
Laser-induced forward transfer is a promising method for 3D printing of various materials, including metals. The ejection mechanism is complex and depends strongly on the experimental parameters, such as laser fluence and donor layer thickness. However, the process can be categorized by the physical condition of the ejected material, i.e., the donor layer is transferred in liquid phase or the material is transferred as a 'pellet' in solid phase. Currently, solid-phase transfer faces several problems. Large shearing forces, occurring at the pellet perimeter during transfer, limit the similarity between the desired pellet shape and the deposited pellet shape. Furthermore, the deposited pellet may be surrounded by debris particles formed by undesired transferred donor material. This work introduces a novel approach for laser-induced forward transfer of variable shaped solid-phase pellets. A liquidcrystal spatial light modulator (SLM) is used to apply grayscale intensity modulation to an incident laser beam to shape the intensity profile. Optimized beams consist of a high fluence perimeter around an interior characterized by a lower fluence level. These beams are used successfully to transfer solid-phase pellets out of a 100-nm Au donor layer using a single laser pulse. The flexibility of the SLM allows a variable desired pellet shape. The shapes of the resulting deposited pellets show a high degree of similarity to the desired shapes. Debris-free deposited pellets are achieved
\end{abstract}

R. Pohl

r.pohl@utwente.nl

1 Chair of Applied Laser Technology, Laboratory of Mechanical Automation and Mechatronics, Faculty of Engineering Technology, University of Twente, Enschede, The Netherlands by pre-machining the donor layer, prior to the transfer, using a double-pulse process.

\section{Introduction}

Laser-induced forward transfer (commonly abbreviated to LIFT) is a direct-write technique that allows the selective transfer of many materials on a micrometer scale [1-5], including liquids [6] and living cells [7, 8]. In the LIFT process, a thin film serves as a donor material that is to be transferred, which is referred to as the donor layer, see Fig. 1. It is supported by a carrier substrate that is transparent to the incident laser wavelength [9]. A pulsed laser is focused on the carrier-donor interface to induce the necessary energy to transfer the donor material onto a receiving substrate, referred to as the receiver. The latter is separated from the donor with a typical gap size in the order of $0-100 \mu \mathrm{m}$.

Recent investigations on the ejection mechanism of various donor layer thicknesses indicate a complex ejection mechanism, which strongly depends on the experimental conditions, such as laser fluence [10]. However, the transfer process can be categorized into broadly two classes, analog to the physical condition of the ejected donor, i.e., transfer of the donor either in liquid phase or solid phase, as illustrated in Fig. 1. For liquid-phase transfer, the irradiated part of the donor layer is melted by the absorbed laser energy to form droplets with dimensions smaller than the diameter of the focal spot $[11,12]$. In the case of solid-phase transfer, the ablation of a thin part of the donor layer near the carrier-donor interface [13] results in the delamination of the donor layer from the carrier substrate. Alternatively, thermally induced stress waves inside the donor layer [14] cause this delamination. The 


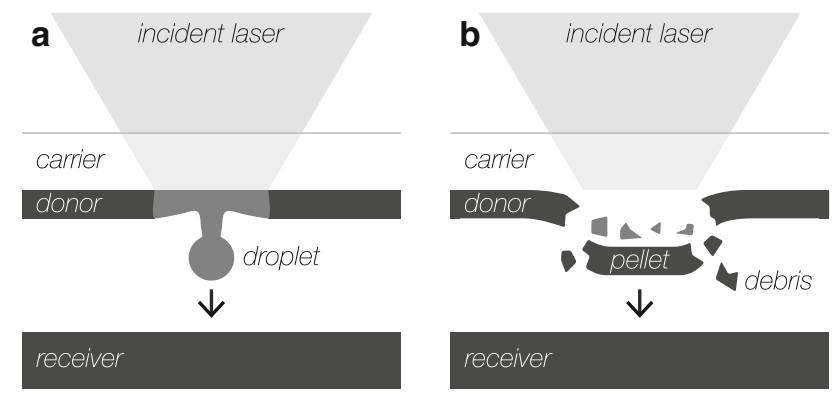

Fig. 1 Schematic representation of LIFT adapted from [15], where the focused laser a melts the donor layer resulting in droplet formation [11, 12] or $\mathbf{b}$ transfers a solid-phase 'pellet' by either ablating a thin part of the donor layer [13] or thermally inducing stress waves inside the donor layer [14]

part of the donor layer that is transferred in solid phase is usually referred to as a 'pellet.'

This paper aims to achieve fast, solid-phase pellet transfer of thin donor layers of variable shapes. Such a transfer technique can be used in applications that require the donor material to remain intact during transfer. Clearly, the first class as described above is not suitable for those applications as the donor material is molten in the process. However, also the second class has drawbacks that complicate pellet transfer in variable shapes:

1. The ejection and transfer of a pellet are typically so 'violent' that often the pellet shatters or even disintegrates.

2. Large shearing forces, occurring at the pellet perimeter during transfer, limit the similarity between the desired pellet shape and the deposited pellet shape.

3. The pellet shape is directly related to the spatial intensity profile of the laser beam, referred to as the beam shape. Variable pellet shapes therefore require spatially shaped beams.

4. Thin donor layers are easily melted; to achieve solidphase pellets, these pellets should be transferred as a result of the thermally induced stress waves studied in [14].

Previous work has attempted to solve these issues. Tolbert et al. [16] introduced a dynamic release layer (DRL) located between the carrier and the donor material, thereby protecting the latter during transfer. Laser decal transfer (LDT), investigated by Piqué et al. [17], uses a viscous suspension of metallic nanoparticles as the donor layer. This so-called nano-ink or nano-paste is irradiated by a flattopped laser beam, using aperture masks to achieve limited flexibility in pellet shape. Auyeung et al. [18, 19] improved LDT by using a digital micro-mirror device (DMD) for spatial beam shaping. DMDs are able to modulate the intensity of the incident laser beam in a binary fashion (on or off) on a per-pixel basis and are used to vary the pellet shape on demand. Ballistic laser-assisted solid transfer
(BLAST), as demonstrated by Banks et al. [13], uses the laser outside its focal plane to achieve beam shapes with a higher intensity perimeter that directly weakens the shearing areas of the donor layer. A focused ion beam (FIB) technique is employed by Kaur et al. [20] to premachine the donor layer in the desired pellet shape. The LIFT process is then used to transfer only the pre-machined part of the donor layer. Recently, Rapp et al. [21] demonstrated the advantages of mask-based smart beam shaping (SBS) on the transfer of PEDOT: PSS pixels, using optimized beam shapes with overexposed perimeters. Though this beam shaping method in principle allows for a variety of beam shapes, it is limited in the flexibility due to the time-consuming mask preparation and the lack of realtime modulation of the desired beam shape.

Neither of these approaches solve all the problems mentioned above. While DRL-LIFT protects the pellet from damage during the LIFT process, it does not address the shearing forces that limit the fidelity of the pellet shape. In contrast, LDT has been used to achieve great flexibility in pellet shape, but this approach is not suitable for applications that require truly solid donor material. BLAST allows solid donor material, but it is limited to mostly circular pellet shapes. The pre-machining step for FIB is time-consuming and therefore costly, although it has the advantage that it provides flexibility in pellet shape. The mask-based SBS allows for flexible beam shapes, but is lacking in real-time flexibility due to the working principle of the mask projection.

This work introduces a novel approach using a liquidcrystal spatial light modulator (SLM) in the LIFT process to achieve solid-phase pellet transfer of variable shapes. In contrast to DMDs that only perform binary intensity modulation, a SLM offers full grayscale intensity modulation when used in a cross-polarization setup [22, 23]. The details of the setup are discussed in Sect. 2. Section 3 provides a proof of concept in the form of successfully transferred solid-phase pellets of varying shapes from 100-nm-thick Au donor layers. Inspired by BLAST [13] and the LIFT of pre-machined donor layers [20], single- and double-pulse experiments with modulated laser beams according to the desired pellet shapes are presented. In both cases, the transfer of solid pellets with the desired shape is achieved. However, the single-pulse ejection suffers from sporadic contamination, and clean ejection is demonstrated only for the double-pulse ejection process.

\section{Experimental setup}

The following two subsections provide a detailed explanation of the SLM beam shaping setup and the preparation of the donor and receiver samples that are used in this 
work. To this end, the principle of operation of the beam shaping setup is described by three steps: irradiation of the SLM with the laser beam, modulation of the intensity profile through polarization-based phase modulation 'on a per-pixel basis,' and imaging of that profile onto the sample through an infinity optical microscope.

\subsection{SLM beam shaping}

Figure 2 shows the cross-polarization beam shaping setup employed in this paper. A picosecond laser (TRUMPF TruMicro 5050 with a frequency doubler, $\lambda=515 \mathrm{~nm}$ ) is used at a pulse repetition rate of $40 \mathrm{~Hz}$. The pulse duration of this source is specified to be $6.7 \mathrm{ps}$ full duration at half maximum. The unfocused beam diameter is approximately $6 \mathrm{~mm}$, and the light is linearly polarized in the vertical direction. The polarizing beam splitter A (Thorlabs CM1PBS251) directs the beam toward the SLM (Hamamatsu X10468-02 [24]).

The SLM consists of $800 \times 600$ pixels on an active area of 16 by $12 \mathrm{~mm}$, resulting in a pixel size of $20 \times 20 \mu \mathrm{m}$. Each pixel can be controlled individually on an 8 bit grayscale range. The birefringence effect of the crystals induces a wavelength-dependent phase retardance in the horizontal component of the incoming beam with respect to the SLM, which is mounted at an angle of $45^{\circ}$. Software was developed to control the grayscale pattern of the SLM, which allows for full control of the status of polarization on the per-pixel basis. Light reflected by the SLM passes through the polarizing beam splitter A a second time. In this case, the splitter transmits only the horizontally polarized component of the laser beam and ultimately allows for the full intensity modulation of the laser beam.

The surface of the SLM, i.e., the shaped laser beam, is imaged onto the carrier-donor interface using a demagnifying microscope setup. The microscope consists of an infinity-corrected tube lens (Thorlabs ITL200, $f=200 \mathrm{~mm}$ ) in combination with a $100 \times$ infinity-corrected long-working distance microscope objective (Mitutoyo Plan Apo \#46-147). The latter is mounted on a translation stage (Newport 562-XYZ) for positioning of the focus location relative to the carrier-donor interface. The spatial resolution of this setup is determined by the resolving power of the microscope objective, specified to be $0.4 \mu \mathrm{m}$. However, due to aberrations caused by the influence of the carrier and due to the optical components placed into the beam path, this diffraction-limited resolution will not be achieved, but is provided as the best-case scenario.

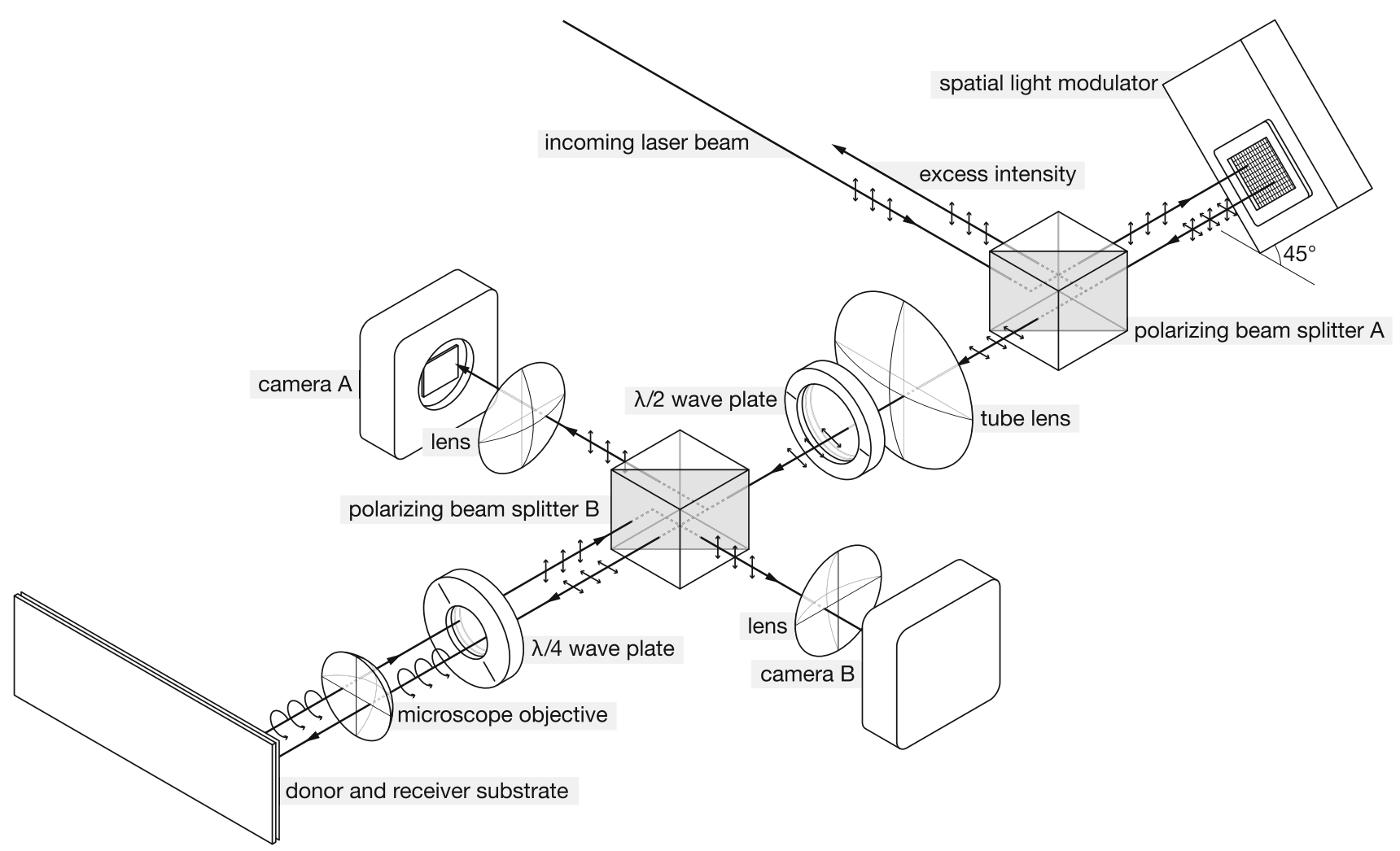

Fig. 2 Schematic of the experimental setup for solid-phase LIFT. Only to guide the readers eye, the different 'arms' of the laser beam are offseted with respect to the optical axis to allow the indication of the different polarization states 
For inline profiling of the beam shape, a half-wave plate (Thorlabs WPH10M-514) on a rotatable mount is placed between the tube lens and the microscope objective. Polarizing beam splitter B (Thorlabs CM1-PBS251) deflects an adjustable part of the intensity of the beam through a lens (Thorlabs LA1509-A, $f=100 \mathrm{~mm}$ ) on camera A (Thorlabs DCC1545M). This camera captures a high-dynamic range image to measure a single beam profile on the SLM. These images are first used to calibrate the SLM in order to correct for any deformations of its surface. The resulting minimum and maximum intensity profiles are shown in Fig. 3, where the maximum profile contains a factor 60 more energy than the minimum profile. At the wavelength of $515 \mathrm{~nm}$, the one $\pi$ phase difference between minimum and maximum intensity corresponds to a graylevel change of 47 on the SLM, limiting the intensity resolution to some extent. The interference pattern observable in the maximum intensity profile (shown in Fig. 3b, c) is likely attributed to 'ghost reflections' at the interfaces (beam path SLM to Camera A) of the polarizing beam splitters and mainly attributed to the performance of the broad band anti-reflection coatings that are applied on each interface.

The desired beam shape is fitted within the maximum intensity profile as shown in Fig. 3c, and the required intensity modulation is calculated by the software in an iterative approach. Using a first-order approximation of the relation between gray level and phase retardance, the initial pattern of the SLM is calculated. A proportional feedback controller, inspired by Liang et al. [25], is applied that iteratively improves the required phase pattern by comparing the desired beam shape to the beam shape measured by camera A. Figure 4 shows this measured beam shape after several iterations of the feedback loop for an arbitrary desired beam shape. Depending on the desired beam shape, the reshaped laser beam contains up to $50 \%$ of the energy of the laser beam incident on the SLM.
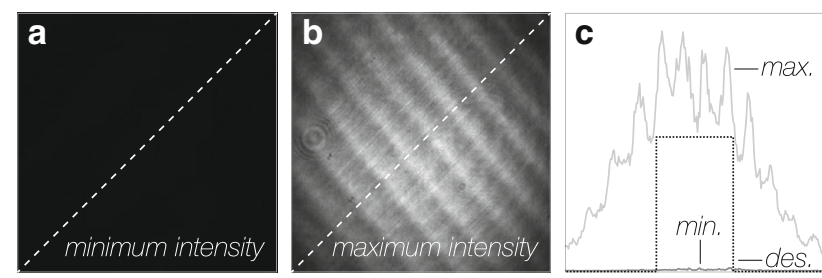

Fig. 3 Laser beam profiles captured by camera A after, respectively, a minimizing and $\mathbf{b}$ maximizing the intensity using the SLM. The grayscale level in these images is a measure for the intensity. The intensity on the dashed lines in profiles $\mathbf{a}$ and $\mathbf{b}$ is plotted together with a desired (flat-topped) beam shape in graph c. The latter is fitted within the maximum intensity; depending on the beam shape, energy efficiencies of up to $50 \%$ can be achieved. In both, profile $\mathbf{b}$ and graph c interference fringes caused by the polarizing beam splitters can be observed
Camera B (Thorlabs DCC1545M) captures light that is reflected from the carrier-donor interface back through the microscope objective. To this end, a quarter-wave plate (Thorlabs WPDM05M-1064H-532Q) is placed between polarizing beam splitter $\mathrm{B}$ and the objective. A lens (Thorlabs LA1509-A $f=100 \mathrm{~mm}$ ) is used to image the carrier-donor interface onto the camera. Being able to view the surface of the donor inline and in real-time brings two major advantages: It allows the focusing of the beam profile on the carrier-donor interface to be adjusted optically, and it easily allows inspection of the donor after each pulse. For the latter, an additional (cold-) light source was used to illuminate the donor surface.

\subsection{Sample preparation and experimental methods}

The donor carrier slide is a $60 \times 24 \times 0.13 \mathrm{~mm}$ hydro-lytic class 1 glass slide (MENZEL-Gläser BB024060A1). A 100-nm donor layer of $\mathrm{Au}$ is deposited on this slide using magnetron sputtering (sputter rate: $23 \mathrm{~nm} / \mathrm{min}$ ). These thin glass slides are chosen to minimize the influence of spherical aberrations leading to a deterioration of the laser beam shapes, as illustrated in Fig. 5. In preparation for the deposition of the donor material, the glasses are first ultrasonically cleaned using isopropanol, ethanol and acetone. They are then washed in a bath of $99 \%$ nitric acid for ten minutes, followed by a quick dump rinse. Prior to sputtering, the slides are baked at $120^{\circ} \mathrm{C}$ for ten minutes to evaporate any remaining water.

The receiver is based on a standard $76 \times 26 \times 1 \mathrm{~mm}$ microscope slide on which a 400-nm Au coating is deposited using the same sputter technique used to create the donor layer. The same material is used for both the donor and receiver layers to optimize the adhesion of the transferred solid-phase pellets to the receiver. The donor and receiver are stacked with the coated sides parallel and facing each other. The separation between the donor and the receiver is set to $50 \mu \mathrm{m}$, using thin metal foils placed in between. This stack is placed on a XYZ translation stage (Thorlabs PT3) that is partially motorized using two actuators (Thorlabs Z825B). The translation is controlled and synchronized with the release of single laser pulses ensuring proper donor refreshment during processing.

A confocal microscope (Keyence VK 9700) is used to characterize the donor and receiver after the experiments.

\section{Results and discussion}

The experimental setup is used for two types of experiments, using either a single-pulse process or doublepulse process to achieve solid-phase pellet transfer. For the first experiment, a single-pulse ejection is investigated. 

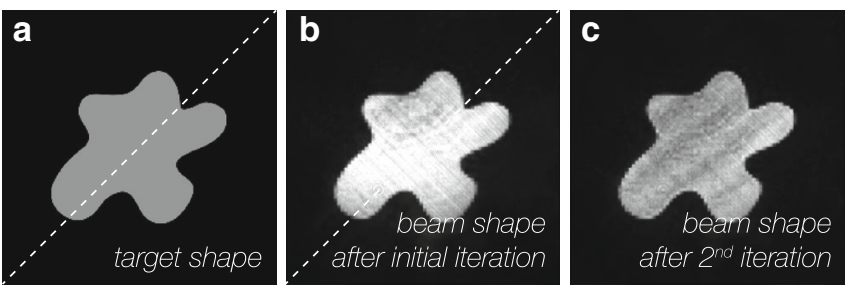

Fig. 4 Laser beam profiles captured by camera A after several iterations of the feedback loop. The grayscale level in these images is a measure for the intensity of the beam shape. The desired beam shape is shown in profile $\mathbf{a}$; profiles $\mathbf{b}-\mathbf{e}$ show the beam shape after, respectively, the initial, second, fourth and sixth iteration. The
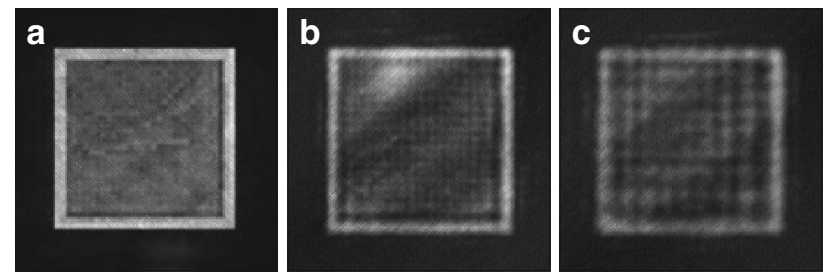

Fig. 5 Laser beam profiles. The grayscale level in these images is a measure for the intensity. Profile $\mathbf{a}$ is captured by camera A directly after it is shaped by the SLM and shows clear distinction between a desired higher fluence perimeter and the lower fluence interior of the beam shape. Both profiles $\mathbf{b}$ and $\mathbf{c}$ are captured by camera B and show the beam shape after it has been reflected by the donor layer. For profile $\mathbf{b}$, a $0.13-\mathrm{mm}$ glass slide is used, while for profile $\mathbf{c}$, a $1.0-\mathrm{mm}$ glass slide is used. The difference in beam shape between profiles $\mathbf{b}$ and $\mathbf{c}$ is caused by the different glass thicknesses

Here, the laser beam is shaped according to a desired pellet shape. The fluence level of the perimeter of the beam is optimized such that it completely melts the irradiated part of the donor layer. In contrast, the fluence level at the interior of the beam is optimized to trigger the ejection process, exploiting thermally induced stresses in the donor layer. The second experiment demonstrates the ejection of solid pellets, using two laser pulses. Therefore, the first pulse is shaped such that it removes an area of the donor layer around the desired pellet shape. The second laser pulse uses a flat-top beam shape to transfer this remaining pellet. The results of these experiments are discussed in the following Sects. 3.1 and 3.2.

\subsection{Single-pulse process}

In order to limit the shearing forces in the donor layer during ejection process, the beam shape is optimized for the desired pellet shape. Therefore, the beam shape resembles the desired pellet shape surrounded by a perimeter at a fluence level that suffices to completely melt the donor layer, similar to [13]. In contrast, the fluence level in the interior of the beam is optimized to prevent melting, while the ejection process is initiated by a thermally induced
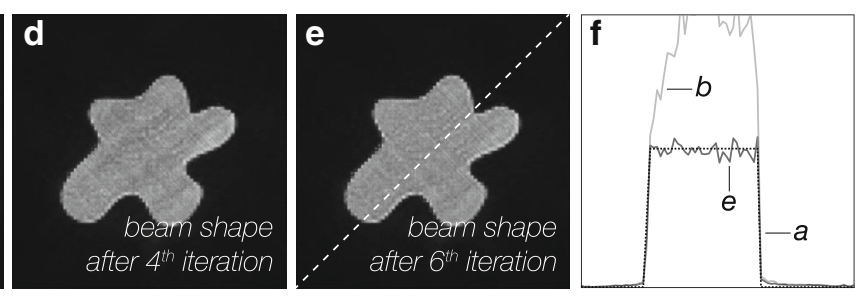

intensity on the indicated lines in profiles $\mathbf{a}, \mathbf{b}$ and $\mathbf{e}$ is plotted in graph f, showing that the beam shape 'converges' to the desired beam shape after six iterations. The RMS error between the desired beam shape and the measured beam shape after the sixth iteration is $8 \%$

stress wave that leads to the ejection of a pellet. It is assumed that the heat flow in the donor is one dimensional: Heat propagates only in the propagation direction of the laser beam. This allows the two fluence levels to be determined sequentially as neither region of the beam shape should affect the other.

The optimal fluence levels are determined using a simple square as the desired pellet shape. The corresponding beam shape including the overexposed perimeter is shown in Fig. 5a. The fluence in the interior is chosen zero to first determine the optimal fluence of the perimeter of the beam shape. Several representative results showing the donor layer are given in Fig. 6. At low fluence levels, such as $0.21 \mathrm{~J} / \mathrm{cm}^{2}$ in Fig. 6a, the perimeter is not completely cut out. The observed asymmetry is attributed to the deterioration of the beam shape inside the carrier slide, as demonstrated in Fig. 5. The use of fluence levels higher than $0.24 \mathrm{~J} / \mathrm{cm}^{2}$ results in a broad cutout, which indicates that the one-dimensional heat flow assumption may not be completely valid. The optimal fluence is found at $0.24 \mathrm{~J} / \mathrm{cm}^{2}$, as this fluence level results in a full cutout of the perimeter with minimum melting. With the perimeter fluence level fixed to $0.24 \mathrm{~J} / \mathrm{cm}^{2}$, the interior fluence level is varied as a percentage of this value to determine the
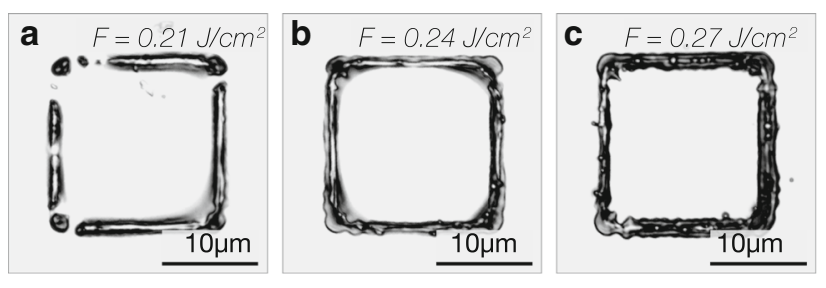

Fig. 6 Donor substrate imaged using confocal microscopy after single-pulse experiments to determine the required border fluence for a square cutout. Sample a is processed with a too low fluence level of $0.21 \mathrm{~J} / \mathrm{cm}^{2}$, resulting in an incomplete cutout, while for $\mathbf{c}$ the fluence level of $0.27 \mathrm{~J} / \mathrm{cm}^{2}$ is too high, resulting in a broad molten border around the desired pellet shape. The fluence level of $0.24 \mathrm{~J} / \mathrm{cm}^{2}$ used for $\mathbf{b}$ is found to be optimal for pellet transferring of 100-nm Au donors 
optimal fluence level to achieve solid-phase pellet transfer. The effect of this fluence level is determined by analyzing the deposited pellets on the receiver, see Fig. 7. From these results, the optimal fluence level of the interior is found to be approximately $20 \%$ of the border fluence or $48 \mathrm{~mJ} / \mathrm{cm}^{2}$. It is clear that the results are quite sensitive to variations in this fluence: Fluence levels smaller than $48 \mathrm{~mJ} / \mathrm{cm}^{2}$ cause too little delamination of the donor and/ or too little thrust to transfer a pellet from the donor to the receiver layer (Fig. 7a), while fluence levels higher than $48 \mathrm{~mJ} / \mathrm{cm}^{2}$ result in the complete melting of the pellet (Fig. 7c).

This is consistent with an estimation of the minimal fluence level $F$ that is required to achieve full melting of the donor layer. Here, the minimal fluence is given by

$F=\frac{d \cdot\left(C_{p} \cdot \Delta T+\rho \cdot L_{m}\right)}{1-R}$,

with the donor layer thickness $d=100 \mathrm{~nm}$, the specific heat capacity $C_{p}=2.5 \cdot 10^{6} \mathrm{~J} /\left(\mathrm{m}^{3} \mathrm{~K}\right)$, density $\rho=19,300$ $\mathrm{kg} / \mathrm{m}^{3}$, latent heat of fusion $L_{m}=0.6 \cdot 10^{5} \mathrm{~J} / \mathrm{kg}$, and reflectivity $R=0.6$ for the given wavelength of $515 \mathrm{~nm}$ wavelength [26, 27]. It is found that the perimeter fluence level of $F=0.24 \mathrm{~J} / \mathrm{cm}^{2}$ is sufficient to melt the donor layer, which is in accordance with the experimental results. Furthermore, it can be shown that experimentally determined optimal fluence level of $\left(F=48 \mathrm{~mJ} / \mathrm{cm}^{2}\right)$ for the interior leads to a temperature raise of $\Delta T \approx 800 \mathrm{~K}$. Therefore, no melting occurs, and it is concluded that the transfer of the pellet is a result of the thermally induced stress waves as studied by Shugaev et al. [14].

To demonstrate the repeatability of this method, a large amount of transfers is made using the optimized fluence levels. Both square and circular desired pellet shapes are chosen, and the receiver is imaged, see Fig. 8. It is noted that these results show that the experiments consistently lead to transferred solid-phase pellets shaped and sized accordingly to the desired pellet shape and corresponding

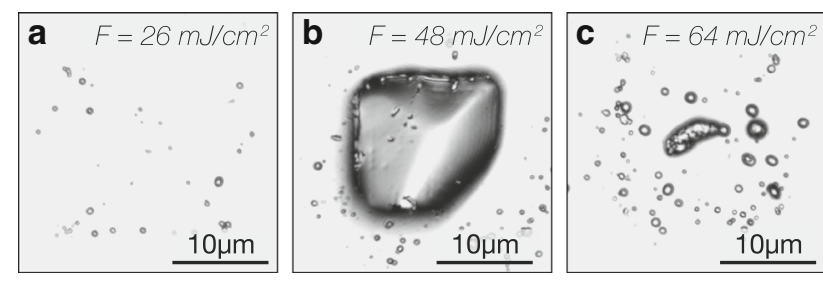

Fig. 7 Receiver substrate imaged using confocal microscopy after single-pulse experiments to determine the required center fluence for transfer of a solid square. Sample a is processed with a too low fluence level of $26 \mathrm{~mJ} / \mathrm{cm}^{2}$, causing the donor layer not to transfer, while for $\mathbf{c}$ the fluence level of $64 \mathrm{~mJ} / \mathrm{cm}^{2}$ is too high resulting in the complete melting of the pellet. The fluence level of $48 \mathrm{~mJ} / \mathrm{cm}^{2}$ used for $\mathbf{b}$ is found to be optimal for 100-nm Au donors beam shape, especially the shape of the circular pellets shows a high similarity to the corresponding desired shape. However, the square pellets have rounded corners, which is a result from the fact that relatively more energy is transferred to the donor layer as two parts of the perimeter meet at a corner. This causes a larger heat up of the donor layer in a corner and therefore slightly more donor material is molten.

Furthermore, it can be observed that all pellets are not flat, but show some consistent deformation. In general, the center of the pellet is raised higher with respect to its perimeter, which may indicate that the center of the pellet was the last area to detach from the donor carrier slide. A possible cause for the latter is some unexpected heat diffusion parallel to the surface of the donor layer. This would cause the areas close to perimeter of the beam shape to heat up faster and delaminate earlier from the carrier, and the observed deformation is caused by the bending of the very thin pellets. The deformation may also be caused by air resistance during the transfer of the pellet from the carrier to the receiver, or a combination of both effects.

The observed bending is expected to have a significant impact on the overall adhesion between the deposited pellets and the receiving substrate. However, in the framework of this article the adhesion was only exploratory studied and reduced to two scenarios, i.e., the deposition on an uncoated glass receiver and the deposition on a gold-coated sample as described in Sect. 2.2. Experiments on depositing solid gold pellets on a glass surface were unsuccessful. That is, the transferred pellets did not stick to the receiver surface but were found at random positions on the receiver, after process. In contrast, the deposition on a gold-coated surface was proven to be successful as the deposited pellets stick to the surface-i.e., robust enough to handle them without special care in a laboratory environment.

Finally, debris is found around the deposited pellet. This is to be expected, as completely melting the perimeter of the shape of the pellet will generate molten donor material that will end up on the receiver layer, even though it can be considered detrimental to the final result.

To further demonstrate the possibilities of the current setup, more elaborate desired pellet shapes were chosen. More specifically, pellets shaped like the characters $U$ and $\mathrm{T}$ (an acronym for the University of Twente) were transferred with the same optimized fluence levels as detailed above. The receiver layer is imaged, see Fig. $8 \mathrm{~h}$, i. The deposited pellets show some deformation, but form a clearly legible $\mathrm{U}$ and $\mathrm{T}$, providing a proof of the flexibility in pellet shapes that this setup provides. Considering the $\mathrm{T}$ as two perpendicular lines, each line is approximately $5 \mu \mathrm{m}$ width and $16 \mu \mathrm{m}$ long. The left part of the $\mathrm{T}$ in Fig. $8 \mathrm{i}$ shows radii of approximately $1 \mu \mathrm{m}$, which represent the resolution of the current setup. However, it should be 

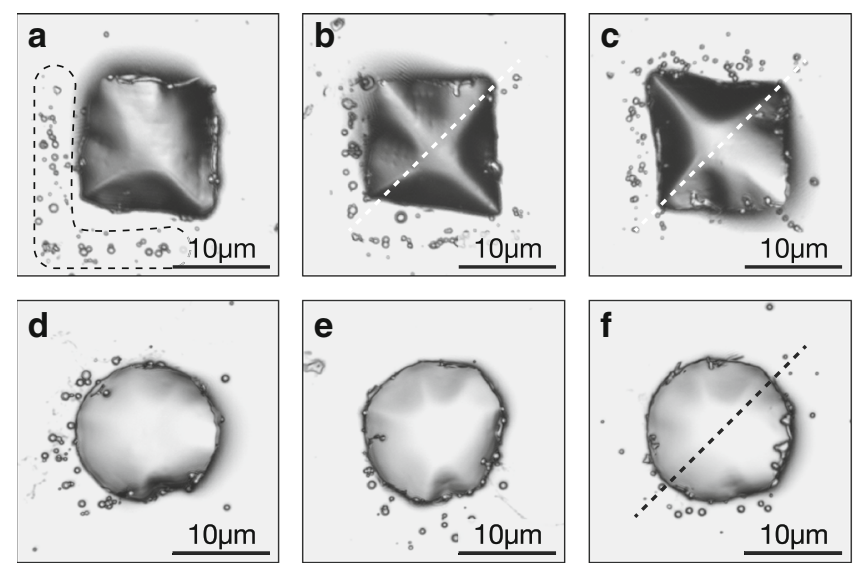

Fig. 8 Receiver substrate imaged using confocal microscopy after single-pulse experiments at optimal fluence levels of $0.24 \mathrm{~J} / \mathrm{cm}^{2}$ for the perimeter and $48 \mathrm{~mJ} / \mathrm{cm}^{2}$ for the interior of the beam shape. Samples a-c correspond to a square desired pellet shape of $19.2 \mu \mathrm{m}$ sides; samples d-f correspond to a circular desired pellet shape $9.6 \mu \mathrm{m}$ in diameter. It should be noted that all pellets show a similar deformation in which the center of each pellet is raised approximately $3 \mu \mathrm{m}$ on average above its perimeter. This is illustrated in graph $\mathbf{g}$ for

mentioned that this feature size may not be the highest resolution of the current setup.

\subsection{Double-pulse process}

To achieve solid-phase pellet transfer without debris, the cutout of the perimeter of the desired pellet shape and the actual transfer of the pellet are performed sequentially using two separate laser pulses. The laser beam for the first pulse is shaped such that it removes an area of the donor layer around the desired pellet shape. For this pre-machining step, the receiver layer is removed from the experimental setup to prevent any molten donor material from contaminating it. The perimeter that is cut out is relatively broad compared to the pellet size, with an outer radius of $21.6 \mu \mathrm{m}$ and an inner radius of $7.2 \mu \mathrm{m}$, respectively, see Fig. 9a. This allows for a high tolerance in alignment of the laser beam with the remaining donor material in the center.

The beam shape that is used to transfer the pellet is circular, flat-topped and has a radius that is twice as large as the radius of the pellet, at a fluence level of $51 \mathrm{~mJ} / \mathrm{cm}^{2}$. An example of such a transferred pellet is shown in Fig. 9b, and there is no observable debris around the deposited pellet.

This process requires the use of multiple pulses to achieve solid-phase LIFT, much as like it is proposed in previous work $[13,20]$. The novel technique described in this work, however, is an improvement on either previous approach. It uses a precisely controlled beam shape to provide greater flexibility in and control over the resulting pellet shape. Furthermore, the required number of pulses to transfer the
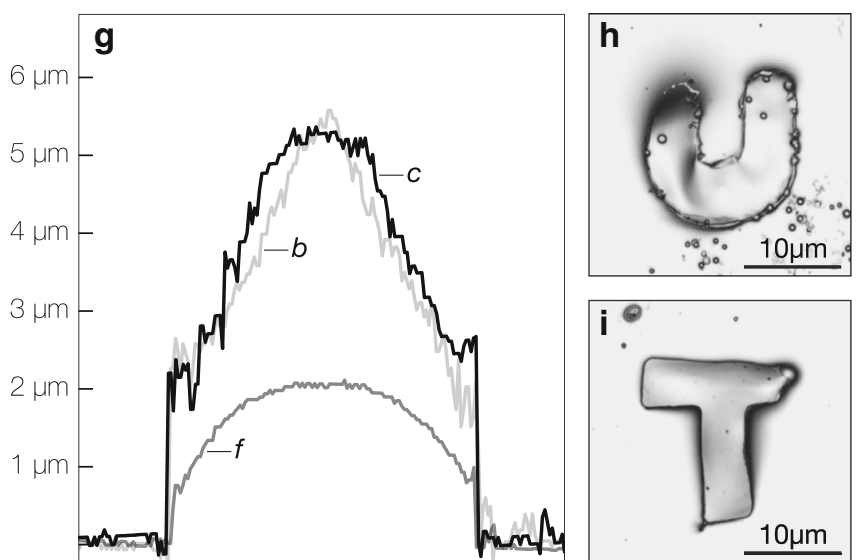

the dashed lines shown on sample $\mathbf{b}, \mathbf{c}$ and $\mathbf{f}$. A likely cause is that the center of the pellets detached slightly later from the donor substrate; then, the edges and the observed deformation are influenced by the bending of the very thin pellets. The debris around the transferred shapes is caused by the melting of the perimeter of the pellet shape. On sample $\mathbf{a}$, this debris is marked with a dashed line. Samples $\mathbf{h}$ and i demonstrate the flexibility in desired pellet shapes achievable
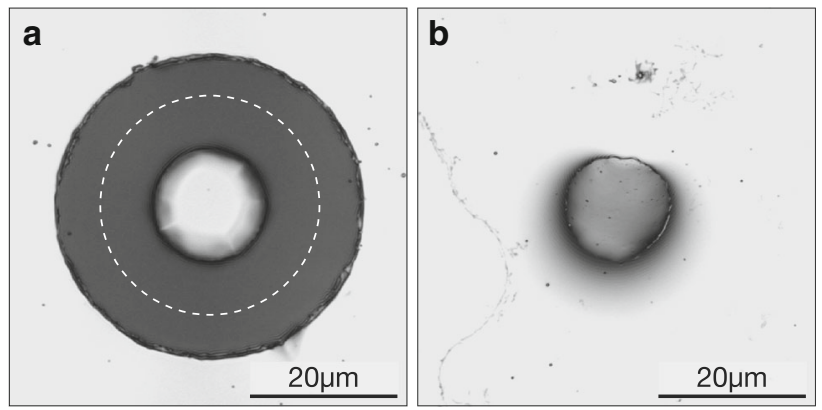

Fig. 9 Donor layer (a) and receiver substrate (b) imaged using confocal microscopy after double-pulse experiments. First, an area around the pellet is removed from the donor layer after which a second laser pulse transfers that pellet intact. The perimeter of the laser beam shape used for transfer is indicated with the dashed line in a

pellet is known a priori (namely exactly two), and only the required fluence level has to be determined. A clear advantage is the implementation using a single setup, without requiring other equipment such as the focused ion beam used by Kaur et al. [20]. This approach gives a real-time flexibility in pellet shape; however, pre-machining can of course still be performed by some other donor preparation techniques.

\section{Conclusion}

This work presented a novel approach using a liquidcrystal spatial light modulator (SLM) in the LIFT process to achieve solid-phase pellet transfer of variable shapes. To this end, intensity profiles consisting of a low fluence interior surrounded by a high fluence perimeter were 
investigated. The optimal laser fluence levels, to achieve solid transfer of an 100-nm-thick gold layer, were experimentally determined and found to be $F=0.24 \mathrm{~J} / \mathrm{cm}^{2}$ and $F=48 \mathrm{~mJ} / \mathrm{cm}^{2}$ for the interior and the perimeter, respectively. Aiming on the transfer of square- and circularshaped pellets, the laser beam was shaped accordingly, and many solid-phase pellets were successfully deposited using the determined optimal fluence levels. Though undesired debris was frequently observed, the shape of these deposited pellets was found to match the desired pellet shapes closely. More complex shapes like the characters $\mathrm{U}$ and $\mathrm{T}$ were successfully deposited and provided as a proof for the flexibility in shape and the spatial resolution that can be obtained with this beam shaping method. In order to transfer and deposit pellets without any debris, a doublepulse process was employed. This approach was proven successful as debris was completely absent at the resulting deposited pellets. Hence, it is concluded that this approach of laser-induced forward transfer shows great potential for debris-free solid transfer of micrometer-sized 2D shapes of thin films.

Open Access This article is distributed under the terms of the Creative Commons Attribution 4.0 International License (http:// creativecommons.org/licenses/by/4.0/), which permits unrestricted use, distribution, and reproduction in any medium, provided you give appropriate credit to the original author(s) and the source, provide a link to the Creative Commons license, and indicate if changes were made.

\section{References}

1. I. Zergioti, S. Mailis, N.A. Vainos, P. Papakonstantinou, C. Kalpouzos, C.P. Grigoropoulos, C. Fotakis, Microdeposition of metal and oxide structures using ultrashort laser pulses. Appl. Phys. A 66(5), 579-582 (1998)

2. D. Toet, P.M. Smith, T.W. Sigmon, M.O. Thompson, Experimental and numerical investigations of a hydrogen-assisted laser-induced materials transfer procedure. J. Appl. Phys. 87(7), 3537-3546 (2000)

3. L. Yang, C. Wang, X. Ni, Z. Wang, W. Jia, L. Chai, Microdroplet deposition of copper film by femtosecond laser-induced forward transfer. Appl. Phys. Lett. 89(16), 161110 (2006)

4. E. Fogarassy, C. Fuchs, F. Kerherve, G. Hauchecorne, J. Perriere, Laser-induced forward transfer of high-Tc $\mathrm{YBaCuO}$ and $\mathrm{BiSr}-$ $\mathrm{CaCuO}$ superconducting thin films. J. Appl. Phys. 66(1), 457-459 (1989)

5. V. Dinca, E. Kasotakis, J. Catherine, A. Mourka, A. Mitraki, A. Popescu, M. Dinescu, M. Farsari, C. Fotakis, Development of peptide-based patterns by laser transfer. Appl. Surf. Sci. 254(4), 1160-1163 (2007)

6. M. Duocastella, J.M. Fernndez-Pradas, P. Serra, J.L. Morenza, Jet formation in the laser forward transfer of liquids. Appl. Phys. A 93, 453-456 (2008a)

7. B. Hopp, T. Smausz, N. Kresz, N. Barna, Z. Bor, L. Kolozsvári, D.B. Chrisey, A. Szabó, A. Nógrádi, Survival and proliferative ability of various living cell types after laser-induced forward transfer. Tissue Eng. 11(11-12), 1817-1823 (2005)
8. M. Duocastella, J.M. Fernández-Pradas, J. Domínguez, P. Serra, J.L. Morenza, Printing biological solutions through laser-induced forward transfer. Appl. Phys. A 93(4), 941-945 (2008b). ISSN $0947-8396$

9. J. Bohandy, B.F. Kim, F.J. Adrian, Metal deposition from a supported metal film using an excimer laser. J. Appl. Phys. 60(4), 1538-1539 (1986)

10. R. Pohl et al., Ejection regimes in picosecond laser-induced forward transfer of metals. Phys. Rev. Appl. 3(2), 024001 (2015)

11. D.P. Banks, C. Grivas, J.D. Mills, R.W. Eason, I. Zergioti, Nanodroplets deposited in microarrays by femtosecond Ti: sapphire laser-induced forward transfer. Appl. Phys. Lett. 89(19), 193107 (2006)

12. D.A. Willis, V. Grosu, Microdroplet deposition by laser-induced forward transfer. Appl. Phys. Lett. 86(24), 244103 (2005)

13. D.P. Banks, C. Grivas, I. Zergioti, R.W. Eason, Ballistic laserassisted solid transfer (BLAST) from a thin film precursor. Opt. Express 16(5), 3249-3254 (2008)

14. M.V. Shugaev, N.M. Bulgakova, Thermodynamic and stress analysis of laser-induced forward transfer of metals. Appl. Phys. A 101(1), 103-109 (2010)

15. D.P. Banks, Femtosecond laser induced forward transfer techniques for the deposition of nanoscale, intact, and solid-phase material. PhD thesis, University of Southampton (2008)

16. W.A. Tolbert, I.-Y. Sandy Lee, M.M. Doxtader, E.W. Ellis, D.D. Dlott, High-speed color imaging by laser ablation transfer with a dynamic release layer: fundamental mechanisms. J. Imaging Sci. Technol. 37(4), 411-421 (1993)

17. A. Piqué, R.C.Y. Auyeung, H. Kim, K. M. Metkus, S.A. Mathews, Digital microfabrication by laser decal transfer. J. Laser Micro Nanoeng. 3(3), 163-168 (2008)

18. R.C.Y. Auyeung, H. Kim, N.A. Charipar, A.J. Birnbaum, S.A. Mathews, A. Piqué, Laser forward transfer based on a spatial light modulator. Appl. Phys. A 102(1), 21-26 (2011)

19. R.C.Y. Auyeung, H. Kim, S. Mathews, A. Piqué, Laser forward transfer using structured light. Opt. Express 23(1), 422-430 (2015)

20. K.S. Kaur, M. Feinaeugle, D.P. Banks, J.Y. Ou, F. Di Pietrantonio, E. Verona, C.L. Sones, R.W. Eason, Laser-induced forward transfer of focussed ion beam pre-machined donors. Appl. Surf. Sci. 257(15), 6650-6653 (2011)

21. L. Rapp, C. Constantinescu, Y. Larmande, A.P. Alloncle, P. Delaporte, Smart beam shaping for the deposition of solid polymeric material by laser forward transfer. Appl. Phys. A 117(1), 333-339 (2014). ISSN 0947-8396

22. J.A. Davis, D.E. McNamara, D.M. Cottrell, T. Sonehara, Twodimensional polarization encoding with a phase-only liquidcrystal spatial light modulator. Appl. Opt. 39(10), 1549-1554 (2000)

23. D. Liu, W. Perrie, L. Mellor, Z. Kuang, E. Fearon, S. Edwardson, G. Dearden, K. Watkins, Picosecond laser beam shaping using a spatial light modulator. In ICALEO, pp. 735-738. ICALEO (2012)

24. LCOS-SLM (Liquid Crystal on Silicon - Spatial Light Modulator). Hamamatsu Photonics, 1 2014. KACC1172E11

25. J. Liang, R.N. Kohn Jr, M.F. Becker, D.J. Heinzen et al., Highprecision laser beam shaping using a binary-amplitude spatial light modulator. Appl. Opt. 49(8), 1323-1330 (2010)

26. D. Bäuerle, Laser Processing and Chemistry, vol. 3 (Springer, Berlin, 2000)

27. A.D. Rakic, A.B. Djurišic, J.M. Elazar, M.L. Majewski, Optical properties of metallic films for vertical-cavity optoelectronic devices. Appl. Opt. 37(22), 5271-5283 (1998) 